\title{
ASYMPTOTIC ANALYSIS OF A QUEUEING SYSTEM WITH A TWO-DIMENSIONAL STATE SPACE
}

\author{
J. P. C. BLANC, ${ }^{*}$ Centre for Mathematics and Computer Science, Amsterdam
}

\begin{abstract}
A technique is developed for the analysis of the asymptotic behaviour in the long run of queueing systems with two waiting lines. The generating function of the time-dependent joint queue-length distribution is obtained with the aid of the theory of boundary value problems of the Riemann-Hilbert type and by introducing a conformal mapping of the unit disk onto a given domain. In the asymptotic analysis an extensive use is made of theorems on the boundary behaviour of such conformal mappings.
\end{abstract}

CONFORMAL MAPPING; RELAXATION TIME

\section{Introduction}

In the performance analysis of computer systems queueing models are frequently encountered which require a two- or more dimensional state space for their description. The mathematical analysis of such models is rather inaccessible. Only recently fairly general techniques have been developed for the two-dimensional case, cf. [5], [6], [4]. The basic idea behind these techniques is the transformation of the functional equation from which the bivariate generating function of the joint queue-length distribution has to be determined into boundary-value problems of the Riemann-Hilbert type. These currently available techniques lead to results concerning the stationary as well as the time-dependent distributions of the stochastic processes involved.

The main goal of the present paper is the development of a technique for the asymptotic analysis as time $\rightarrow \infty$ of the time-dependent distributions for queueing models which need a two-dimensional state space for their description. This asymptotic analysis is important for several reasons. Firstly, for establishing the necessary and sufficient conditions for the process to be ergodic. Quite often it is not even simple to guess these conditions on the basis of intuitive arguments; consider e.g. the coupled processor model in [5] and the ALOHA satellite packet

\footnotetext{
Received 2 December 1982; revision received 23 December 1983.

* Present address: Department of Mathematics and Informatics, Delft University of Technology, Julianalaan 132, 2628 BL, Delft, The Netherlands.
} 
switching models in [9]. Secondly, it is of great practical importance to obtain information (in the ergodic case) about the time a system needs to reach a situation in which it can be considered to be in stochastic equilibrium, after the start of, or a disturbance in, the system. As a measure for this time the concept of relaxation time was introduced for the one-dimensional case (see e.g. [3]). To determine the relaxation time of a queueing system the second term of the asymptotic expansion of the queue-length distribution is needed.

The ideas and techniques which are required in the construction of such an asymptotic analysis will be elucidated by describing them for a particular model, closely related to the $M / G / 1$ model with alternating service discipline studied in [4], §III.2. From a practical point of view this model is a little artificial, but its analysis brings clearly forward all the essential points also encountered in the analysis of more complicated models. In forthcoming papers, e.g. [2], the technique developed in this paper will be used for the asymptotic analysis of the time-dependent behaviour of two-node Jackson networks, cf. [8]. The ultimate goal of our research concerns the asymptotic analysis of time-dependent phenomena in many-node queueing networks.

The organization of this paper is as follows. The queueing model to be investigated will be described in Section 2. It is an $M / G / 1$ model with two types of customers and a paired service discipline, i.e. two customers of different type are served simultaneously. Section 3 is devoted to the discussion of the embedded queue-length process at departure instants; in Section 4 the bivariate generating function of the queue-length process in continuous time will be derived. Section 5 is concerned with the asymptotic analysis of a characteristic integral. The results are used in Section 6 to obtain the conditions for ergodicity and to investigate the relaxation time of the process. Section 7 contains remarks on the evaluation of the conformal mapping which occurs in the solution of the boundary-value problem, on the waiting-time distribution, and on a simple generalization of the queueing model.

\section{The model; definitions}

The following queueing model will be considered. Customers arrive at a single service facility according to a Poisson process with mean interarrival time $\alpha$. With equal probabilities an arriving customer is of type 1 or of type 2 . An arriving customer who finds the system empty is immediately taken into service; otherwise he joins queue 1 or 2 depending on his type. As soon as a service has been completed, a new service is started if any customers are present. If after the completion of a service two customers of different type are present, then they are served simultaneously. If after the completion of a service the customer population consists of one type, then a single customer is admitted to service. In 
each queue customers are served in order of their arrival. Successive service times are independent random variables with a common distribution function $B(\tau)$, for paired services as well as for individual services.

Let $\boldsymbol{y}_{j}(t), t \geqq 0, j=1,2$, be the number of type $j$ customers present in the system at time $t$, and let $\boldsymbol{y}_{j}(0)=0$. Our aim is to study the time-dependent behaviour of the process $\left\{\left(y_{1}(t), y_{2}(t)\right), t \geqq 0\right\}$, especially its asymptotic behaviour as $t \rightarrow \infty$. In order to obtain the distribution of this process first the embedded process at departure instants will be analysed, and then the continuous-time distribution will be derived with the aid of renewal functions, in analogy with the analysis of the standard $M / G / 1$ model, cf. [3], \$II.4.3. Denote by $\boldsymbol{d}_{n}, n=$ $0,1, \cdots$, the $n$th departure instant, and by $x_{j}(n), n=0,1, \cdots, j=1,2$, the number of type $j$ customers left behind in the system at the $n$th departure instant. Let $d_{0}=0, \boldsymbol{x}_{1}(0)=\boldsymbol{x}_{2}(0)=0$, in agreement with the assumption that the process starts at $t=0$ with an empty system. It is readily seen that the process $\left\{\left(\boldsymbol{x}_{1}(n), \boldsymbol{x}_{2}(n), \boldsymbol{d}_{n}\right), n=0,1, \cdots\right\}$ is an embedded Markov chain which is irreducible and aperiodic. This Markov chain was analysed in [1]. The results are summarized in the next section (see also Section 7). For the analysis of the queueing system the following functions and quantities are defined: for $|r|<1$, $\left|z_{1}\right| \leqq 1,\left|z_{2}\right| \leqq 1, \operatorname{Re} \rho \geqq 0$,

$$
\begin{gathered}
\Phi\left(r ; z_{1}, z_{2}, \rho\right):=\sum_{n=0}^{\infty} r^{n} E\left\{z_{1^{x_{1}(n)}} z_{2^{2^{2}}}^{x^{(n)}} \exp \left(-\rho d_{n}\right)\right\}, \\
\Psi\left(\rho ; z_{1}, z_{2}\right):=\int_{0}^{\infty} e^{-\rho t} E\left\{z_{1}^{y_{1}^{(t)}} z_{2^{y}}^{y(t)}\right\} d t ; \\
\beta(\zeta):=\int_{0}^{\infty} e^{-\zeta \tau} d B(\tau), \quad \operatorname{Re} \zeta \geqq 0 ; \\
\beta_{k}:=\int_{0}^{\infty} \tau^{k} d B(\tau), \quad k=1,2, \cdots ; \\
a:=\beta_{1} / \alpha .
\end{gathered}
$$

It will be assumed that $\beta_{3}<\propto$ (see Remark 1 in Section 6).

\section{The embedded Markov chain}

For the transform (1) the following functional equation can be derived in a similar way as that of the common $M / G / 1$ queueing model, cf. [3], §II.4.3: for $|r|<1,\left|z_{1}\right| \leqq 1,\left|z_{2}\right| \leqq 1, \operatorname{Re} \rho \geqq 0$,

$$
\begin{aligned}
& {\left[z_{1} z_{2}-r \beta\left(\rho+\frac{1-\frac{1}{2} z_{1}-\frac{1}{2} z_{2}}{\alpha}\right)\right] \Phi\left(r ; z_{1}, z_{2}, \rho\right)=z_{1} z_{2}+r \beta\left(\rho+\frac{1-\frac{1}{2} z_{1}-\frac{1}{2} z_{2}}{\alpha}\right)} \\
& \times\left[\left(z_{2}-1\right) \Phi\left(r ; z_{1}, 0, \rho\right)+\left(z_{1}-1\right) \Phi\left(r ; 0, z_{2}, \rho\right)\right. \\
& \left.\quad+\left(1-z_{1}-z_{2}+\frac{z_{1} z_{2}}{1+\alpha \rho}\right) \Phi(r ; 0,0, \rho)\right] .
\end{aligned}
$$


Below we describe briefly how this functional equation can be transformed into a boundary-value problem; for details see [1].

From now on it will be assumed that $r$ and $\rho$ are real, $0<r<1, \rho>0$. In the functional equation (6) let $z_{1}=w, z_{2}=\bar{w}$, and let $w$ be in the set

$$
L(r ; \rho):=\left\{w ;|w|<1,|w|^{2}=r \beta\left(\rho+\frac{1-\operatorname{Re} w}{\alpha}\right)\right\} .
$$

For this choice of $z_{1}$ and $z_{2}$ the generating function $\Phi\left(r ; z_{1}, z_{2}, \rho\right)$ is finite, while its coefficient in the functional equation (6) vanishes; hence the functional equation reduces to: for $w \in L(r ; \rho)$,

(8) $\frac{\Phi(r ; w, 0, \rho)}{1-w}+\frac{\Phi(r ; 0, \bar{w}, \rho)}{1-\bar{w}}=\frac{1}{|1-w|^{2}}+\left[1-\frac{|w|^{2}}{|1-w|^{2}} \frac{\alpha \rho}{1+\alpha \rho}\right] \Phi(r ; 0,0, \rho)$.

From the properties of the Laplace-Stieltjes transform $\beta(\zeta)$, cf. (3), it is readily seen that $L(r ; \rho)$ is a contour (i.e. it is a closed curve which does not intersect itself) which has the real axis as an axis of symmetry. Therefore it is possible to introduce the conformal mapping $g(r ; \rho ; z)$ of the unit disk $|z|<1$ onto the domain $L^{+}(r ; \rho)$, the interior of the contour $L(r ; \rho)$, which is uniquely determined by the conditions (cf. [10], Theorem 1.2, 1.3):

$$
g(r ; \rho ; 0)=0, \quad \frac{\partial}{\partial z} g(r ; \rho ; z)>0, \quad \text { at } z=0 .
$$

By [10], Theorem 2.24, the conformal mapping $g(r ; \rho ; z)$ is continuous in the region $|z| \leqq 1$, and maps the unit circle $|z|=1$ one-to-one onto the contour $L(r ; \rho)$. Moreover, the symmetry of $L(r ; \rho)$ leads to the property: for $|z| \leqq 1$,

$$
g(r ; \rho ; \bar{z})=\overline{g(r ; \rho ; z)} .
$$

By inserting $w=g(r ; \rho ; u),|u|=1$, so that $\bar{w}=g(r ; \rho ; 1 / u)$ by $(10)$, Equation (8) becomes: for $|u|=1$,

$$
\begin{aligned}
& \frac{\Phi(r ; g(r ; \rho ; u), 0, \rho)}{1-g(r ; \rho ; u)}+\frac{\Phi(r ; 0, g(r ; \rho ; 1 / u), \rho)}{1-g(r ; \rho ; 1 / u)} \\
& \quad=\frac{1}{|1-g(r ; \rho ; u)|^{2}}+\left[1-\frac{|g(r ; \rho ; u)|^{2}}{|1-g(r ; \rho ; u)|^{2}} \frac{\alpha \rho}{1+\alpha \rho}\right] \Phi(r ; 0,0, \rho) .
\end{aligned}
$$

Because $L^{+}(r ; \rho) \subset\{w ;|w|<1\}$, the first term at the left-hand side of (11) is regular for $|u|<1$, the second term for $|u|>1$. Hence, relation (11) forms the boundary condition of a coupling problem (or Hilbert problem), cf. [11], §37. It is easily solved by applying the operator

$$
\frac{1}{2 \pi i} \int_{C} \cdots \frac{d u}{u-z}, \quad C:=\{u ;|u|=1\}
$$


on both sides of Equation (11), for $|z|<1$ as well as for $|z|>1$. The last unknown $\Phi(r ; 0,0, \rho)$ is obtained by taking $z=0$. By introducing the inverse conformal mapping $g_{0}(r ; \rho ; w)$ of $g(r ; \rho ; z)$ the functions $\Phi\left(r ; z_{1}, 0, \rho\right)$ and $\Phi\left(r ; 0, z_{2}, \rho\right)$ can be obtained. Substitution of these functions into the functional equation (6) leads to the following result.

Theorem 1. For $0<r<1, \rho \geqq 0, z_{1} \in L^{+}(r ; \rho), z_{2} \in L^{+}(r ; \rho)$,

$$
\begin{aligned}
& \Phi\left(r ; z_{1}, z_{2}, \rho\right)=\left[r \beta\left(\rho+\frac{1-\frac{1}{2} z_{1}-\frac{1}{2} z_{2}}{\alpha}\right)-z_{1} z_{2}\right]^{-1} \\
& \times\left[-z_{1} z_{2}+\frac{\left(1-z_{1}\right)\left(1-z_{2}\right) r \beta\left(\rho+\frac{1-\frac{1}{2} z_{1}-\frac{1}{2} z_{2}}{\alpha}\right)}{1+\frac{\alpha \rho}{2 \pi i} \int_{C} \frac{1}{|1-g(r ; \rho ; u)|^{2}} \frac{d u}{u}}\right. \\
& \times\left\{\left[\frac{\alpha \rho}{\left(1-z_{1}\right)\left(1-z_{2}\right)}-1\right] \frac{1}{2 \pi i} \int_{C} \frac{1}{|1-g(r ; \rho ; u)|^{2}} \frac{d u}{u}\right. \\
& +\frac{1}{2 \pi i} \int_{C} \frac{1}{|1-g(r ; \rho ; u)|^{2}}\left[\frac{1}{u-g_{0}\left(r ; \rho ; z_{1}\right)}\right. \\
& \left.\left.+\frac{1}{u-g_{0}\left(r ; \rho ; z_{2}\right)}\right] d u\right\}
\end{aligned}
$$

Note that the generating function $\Phi\left(r ; z_{1}, z_{2}, \rho\right),|r|<1,\left|z_{1}\right| \leqq 1,\left|z_{2}\right| \leqq 1$, $\operatorname{Re} \rho \geqq 0$, is determined by analytic continuation from the above expression.

\section{The continuous-time process}

By using a relation similar to [3], formula (II.4.45), between the distribution of $\left(y_{1}(t), y_{2}(t)\right), t \geqq 0$, and that of $\left(x_{1}(x), x_{2}(n), d_{n}\right), n=0,1, \cdots$, the following relation between the generating functions $\Psi\left(\rho ; z_{1}, z_{2}\right)$ and $\Phi\left(r ; z_{1}, z_{2}, \rho\right)$ is obtained: for $\left|z_{1}\right| \leqq 1,\left|z_{2}\right| \leqq 1, \operatorname{Re} \rho>0$,

$$
\begin{aligned}
\Psi\left(\rho ; z_{1}, z_{1}\right)= & \frac{\alpha}{1+\alpha \rho} \beta\left(\rho+\frac{\left.1-\frac{1}{2} z_{1}-\frac{1}{2} z_{2}\right)}{\alpha}\right) \Phi(1 ; 0,0, \rho) \\
& +\alpha \frac{1-\beta\left(\rho+\frac{1-\frac{1}{2} z_{1}-\frac{1}{2} z_{2}}{\alpha}\right)}{\alpha \rho+1-\frac{1}{2} z_{1}-\frac{1}{2} z_{2}} \Phi\left(1 ; z_{1}, z_{2}, \rho\right) .
\end{aligned}
$$

Hence, the function $\Psi\left(\rho ; z_{1}, z_{2}\right)$ is also determined by Theorem 1 . This function can also be obtained with the aid of two supplementary variables, see [1], Chapter III. Introducing for real $\rho, \rho>0$, the abbreviations,

$$
\gamma(\rho ; z):=g(1 ; \rho ; z), \quad|z| \leqq 1, \quad \Lambda(\rho):=L(1 ; \rho),
$$

it follows from (13) and Theorem 1 that for $\rho>0$, 


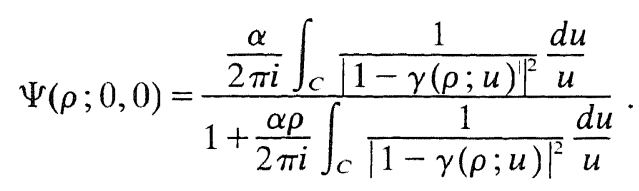

In the next sections the asymptotic behaviour of the process $\left\{\left(\boldsymbol{y}_{1}(t), \boldsymbol{y}_{2}(t)\right), t \geqq 0\right\}$, as $t \rightarrow \infty$ will be studied. Similarly as for the common $M / G / 1$ queueing system it can be proved with the aid of the key renewal theorem, cf. [3], p. 102, p. 246, that the limits

$$
\lim _{t \rightarrow \infty} \operatorname{Pr}\left\{\boldsymbol{y}_{1}(t)=k_{1}, \boldsymbol{y}_{2}(t)=k_{2}\right\}, \quad k_{1}=0,1, \cdots, \quad k_{2}=0,1, \cdots,
$$

exist. Hence, the generating function of this limiting distribution can be obtained from $\Psi\left(\rho ; z_{1}, z_{2}\right)$ with the aid of an Abelian theorem, in particular,

$$
\psi_{0}:=\lim _{t \rightarrow \infty} \operatorname{Pr}\left\{y_{1}(t)=0, y_{2}(t)=0\right\}=\lim _{\rho \downarrow 0} \rho \Psi(\rho ; 0,0) .
$$

\section{Asymptotic analysis}

In order to study the asymptotic behaviour of $\operatorname{Pr}\left\{\boldsymbol{y}_{1}(t)=\boldsymbol{y}_{2}(t)=0\right\}$ as $t \rightarrow \infty$ this section is devoted to the investigation of the limit, cf. (16), (15),

$$
\lim _{\rho \downarrow} \frac{\alpha \rho}{2 \pi i} \int_{C} \frac{1}{|1-\gamma(\rho ; u)|^{2}} \frac{d u}{u}
$$

For the determination of this limit the behaviour of the contour $\Lambda(\rho)$ and of the conformal mapping $\gamma(\rho ; z)$ as $\rho \downarrow 0$ will be considered first. In order to obtain a parametric equation for the contour $\Lambda(\rho)$ the following result is needed.

Lemma 1. For $\rho>0, u \leqq 1$, and for $\rho \geqq 0, u<1$, the equation

$$
\sigma^{2}=\beta\left(\rho+\frac{1-\sigma u}{\alpha}\right) \text {, }
$$

has exactly one root $\sigma=\sigma(\rho ; u)$ on the real interval $0<\sigma<1$. This root $\sigma(\rho ; u)$ is an infinitely differentiable function of $\rho$ and $u$, with

$$
\frac{\partial}{\partial \rho} \sigma(\rho ; u)<0, \quad \frac{\partial}{\partial u} \sigma(\rho ; u)>0, \quad \text { for } \rho>0, u \leqq 1 .
$$

Further,

$$
\begin{gathered}
\sigma(u):=\lim _{\rho \downarrow 0} \sigma(\rho ; u) \begin{cases}=1, & \text { if } u=1, a \leqq 2, \\
\in(0,1), & \text { otherwise; }\end{cases} \\
\sigma^{\prime}(1)=\frac{a}{2-a}, \quad \sigma^{\prime \prime}(1)=\frac{2 a^{2}}{(2-a)^{3}}\left(2 \beta_{2} / \beta_{1}^{2}+1-a\right), \quad \text { if } a<2,
\end{gathered}
$$




$$
\begin{aligned}
& \sigma(u)=1-\sqrt{\frac{2}{2 \beta_{2} / \beta_{1}^{2}-1}} \sqrt{1-u}+O(1-u), \quad u \uparrow 1 \text {, if } a=2 \text {; } \\
& \lim _{\rho \downarrow 0} \frac{\partial}{\partial \rho} \sigma(\rho ; 1)=\frac{-\beta_{1}}{2-a}, \quad \text { if } a<2, \\
& \sigma(\rho ; 1)=1-\sqrt{\frac{\beta_{1} \rho}{2 \beta_{2} / \beta_{1}^{2}-1}}+O(\rho), \quad \rho \downarrow 0, \text { if } a=2 .
\end{aligned}
$$

Proof. The proof is left to the reader. It is very similar to that of the lemma of Takács, cf. [3]. See also [1], Lemma III.4.1.

With the aid of the function $\sigma(\rho ; u)$ the contour $\Lambda(\rho)$ can be described by, cf. (14), (7), (18), for $\rho>0$,

$$
\Lambda(\rho)=\left\{w ; w=\sigma(\rho ; \cos \theta) e^{i \theta},-\pi \leqq \theta \leqq \pi\right\} .
$$

As $\rho \downarrow 0$ the contour $\Lambda(\rho)$ expands, cf. (19), to the contour $\Lambda$ given by:

$$
\Lambda:=\left\{w ; w=\sigma(\cos \theta) e^{i \theta},-\pi \leqq \theta \leqq \pi\right\} .
$$

Lemma 2. The contour $\Lambda(\rho), \rho>0$, possesses a tangent at every point. The contour $\Lambda$ possesses a tangent at every point, except in the case $a=2$ at the point $w=1$; it has then at $w=1$ a corner point with inner angle $\omega \pi$,

$$
\omega \pi:=2 \arctan \sqrt{2 \beta_{2} / \beta_{1}^{2}-1}, \quad \frac{1}{2} \leqq \omega<1 .
$$

Proof. See the appendix.

Next the conformal mapping $\gamma(z)$ of the unit disk $|z|<1$ onto the interior $\Lambda^{+}$ of the contour $\Lambda$, satisfying $\gamma(0)=0, \gamma^{\prime}(0)>0$, cf. (9), (14), is introduced. Because $\Lambda(\rho)$ expands continuously to the contour $\Lambda$ as $\rho \downarrow 0$, cf. (25), (19), it follows by Carathéodory's mapping theorem, cf. [10], Theorem 2.1, that

$$
\lim _{\rho \downarrow 0} \gamma(\rho ; z)=\gamma(z)
$$

uniformly for $|z|<1$; because $\gamma(\rho ; z), \rho>0$, and $\gamma(z)$ are continuous for $|z| \leqq 1$, cf. Section 3 , this limit also holds for $|z|=1$.

Lemma 3. For $\rho>0$ the derivative $(\partial / \partial z) \gamma(\rho ; z)$ is continuous and nonvanishing for $|z| \leqq 1$. The derivative $\gamma^{\prime}(z)$ is continuous and non-vanishing for $|z| \leqq 1$, except in the case $a=2$ at $z=1$. In the case $a<2$, for every $\delta, 0<\delta<1$,

$$
\gamma(z)=1+(z-1) \gamma^{\prime}(1)+O\left(|1-z|^{2-\delta}\right), \quad z \rightarrow 1, \quad|z| \leqq 1 ;
$$

in the case $a=2$ there exist positive constants $N_{1}, N_{2}$, such that

$$
N_{1}|1-z| \leqq|1-\gamma(z)| \leqq N_{2} \sqrt{|1-z|}, \quad|z| \leqq 1 .
$$


Proof. See the appendix.

With the aid of the foregoing lemmas the following theorems on the limit (17) can be proved.

Theorem 2. In the case $a>2$ the limit

$$
\lim _{\rho \downarrow 0} \frac{1}{2 \pi i} \int_{C} \frac{1}{|1-\gamma(\rho ; u)|^{2}} \frac{d u}{u}
$$

is finite; in the case $a=2$ this limit is infinite.

Proof. Because $\gamma(\rho ; u) \in \Lambda(\rho)$ for $|u|=1$, it follows from Lemma 1, cf. (19), (25), (26), that $|\gamma(\rho ; u)|<1$ for $\rho>0,|u| \leqq 1$, and that $|\gamma(u)|<1$ for $|u| \leqq 1$, except in the case $a \leqq 2$ at $u=1$; then $\gamma(1)=\sigma(1)=1$. Hence, in the case $a>2$ the integrand, and therefore also the integral, in (31) remain finite as $\rho \downarrow 0$. Consider further the case $a=2$. The integral in (31) is equal to

$$
\frac{1}{\pi} \int_{0}^{\pi} \frac{d \theta}{\left|1-\gamma\left(\rho ; e^{i \theta}\right)\right|^{2}} \text {. }
$$

From the above it follows that the integrand of this integral remains finite as $\rho \downarrow 0$ for every $\theta$ except $\theta=0$. Lemma 3 implies that there exists a positive $M$ independent of $\theta$ and $\rho$ such that for $\rho>0,0 \leqq \theta \leqq \pi$,

$$
\left|\gamma(\rho ; 1)-\gamma\left(\rho ; e^{i \theta}\right)\right| \leqq M|\theta|^{1 / 2}
$$

This implies the inequality: for $\rho>0,0 \leqq \theta \leqq \pi$,

$$
\left|1-\gamma\left(\rho ; e^{i \theta}\right)\right| \leqq|1-\gamma(\rho ; 1)|+M|\theta|^{1 / 2} .
$$

This inequality leads to the following lower bound for the integral in (32): for $M$ independent of $\rho, \rho>0$,

$$
\begin{aligned}
\frac{1}{\pi} \int_{0}^{\pi} \frac{d \theta}{\left|1-\gamma\left(\rho ; e^{i \theta}\right)\right|^{2}} \geqq \frac{1}{\pi} \int_{0}^{\pi} \frac{d \theta}{\{1-\gamma(\rho ; 1)+M \vee \theta\}^{2}} \\
=\frac{2}{\pi M^{2}}[\log \{1-\gamma(\rho ; 1)+M \vee \pi\}-\log \{1-\gamma(\rho ; 1)\} \\
\\
\left.-\frac{M \vee \pi}{1-\gamma(\rho ; 1)+M \vee \pi}\right]
\end{aligned}
$$

Because $M$ is positive and $\gamma(\rho ; 1) \uparrow 1$ as $\rho \downarrow 0$, it is clear that this lower bound tends to $x$ as $\rho \downarrow 0$. This proves the assertion in the case $a=2$.

Theorem 3.

$$
\lim _{\rho \downarrow 0} \frac{\alpha \rho}{2 \pi i} \int_{C} \frac{1}{|1-\gamma(\rho ; u)|^{2}} \frac{d u}{u}= \begin{cases}0, & \text { if } a=2 \\ \frac{1-\frac{1}{2} a}{a \gamma^{\prime}(1)}, & \text { if } a<2 .\end{cases}
$$


Proof. First consider the case $a=2$. As in the proof of Theorem 2 the integral in (36) is rewritten as (32). From Lemma 3 it follows that for, say, $0<\rho<1$ there exists a positive $K$ independent of $\rho$ and $\theta$ such that for $0 \leqq \theta \leqq \pi$,

$$
\left|\gamma(\rho ; 1)-\gamma\left(\rho ; e^{i \theta}\right)\right| \geqq K|\theta|
$$

Because the point $w=\gamma(\rho ; 1)=\sigma(\rho ; 1)$ is the point on $\Lambda(\rho)$ with the largest absolute value (cf. (25), $\sigma(\rho ; u)$ is for fixed $\rho, \rho>0$, an increasing function of $u$, $u \leqq 1$, cf. (18)), the angle which the line joining the points $\gamma(\rho ; 1)$ and $\gamma\left(\rho ; e^{i \theta}\right)$ makes with the positive direction on the real axis is obtuse. Hence, the cosine rule implies for $\rho>0,0 \leqq \theta \leqq \pi$,

$$
\left|1-\gamma\left(\rho ; e^{i \theta}\right)\right|^{2} \geqq|1-\gamma(\rho ; 1)|^{2}+\left|\gamma(\rho ; 1)-\gamma\left(\rho ; e^{i \theta}\right)\right|^{2} .
$$

From (37), (38), the following upper bound for the integral (32) is obtained: for $0<\rho<1$ and for $K$ independent of $\rho$,

$$
\begin{aligned}
\frac{1}{\pi} \int_{0}^{\pi} \frac{d \theta}{\sqrt{1-\left.\gamma\left(\rho ; e^{i \theta}\right)\right|^{2}}} & \leqq \frac{1}{\pi} \int_{0}^{\pi} \frac{d \theta}{|1-\gamma(\rho ; 1)|^{2}+K^{2} \theta^{2}} \\
& =\frac{1}{\pi K\{1-\gamma(\rho ; 1)\}} \arctan \left[\frac{\pi K}{1-\gamma(\rho ; 1)}\right] .
\end{aligned}
$$

Because $\gamma(\rho ; 1)=\sigma(\rho ; 1)$, cf. (25), it follows from (24) that

$$
\lim _{\rho \downarrow 0} \frac{\rho}{1-\gamma(\rho ; 1)}=0, \quad \lim _{\rho \downarrow 0} \arctan \left[\frac{\pi K}{1-\gamma(\rho ; 1)}\right]=\frac{1}{2} \pi \text {. }
$$

Hence, the upper bound for the integral (32) given in (39), multiplied by $\rho$, vanishes as $\rho \downarrow 0$. This proves the assertion for the case $a=2$.

Next consider the case $a<2$. Because for $\rho>0$ the function $\sigma(\rho ; u)$ is an infinitely differentiable function of $u, u \leqq 1$, cf. Lemma $1, \Lambda(\rho)$ is an analytic contour, cf. [12], p. 186. This implies that the conformal mapping $\gamma(\rho ; z)$ is regular on the boundary $|z|=1$, cf. [12], p. 186, so that it can be continued analytically into a part of the region $|z|>1$. Further, because the derivative $(\partial / \partial z) \gamma(\rho ; z)$ is non-vanishing at $z=1$ by Lemma 3 , and since $\gamma(\rho ; 1) \uparrow 1$ as $\rho \downarrow 0$, it follows (see [1], §II.5 for more details) that for $\rho$ close to 0 there exists a value $u_{0}(\rho)>1$ such that

$$
\gamma\left(\rho ; u_{0}(\rho)\right)=1, \quad \text { and } \quad u_{0}(\rho) \downarrow 1 \quad \text { as } \rho \downarrow 0 .
$$

With this $u_{0}(\rho)$ the integral in (36) is rewritten as: for $\rho$ close to 0 ,

$$
\int_{C} \frac{1}{\sqrt{1-\left.\gamma(\rho ; u)\right|^{2}}} \frac{d u}{u}=\int_{C} K(\rho ; u) \frac{1}{\left\{u-u_{0}(\rho)\right\}\left\{u u_{0}(\rho)-1\right\}} \frac{d u}{u},
$$


here

$$
K(\rho ; u):=\frac{u-u_{0}(\rho)}{1-\gamma(\rho ; u)} \frac{u u_{0}(\rho)-1}{1-\gamma(\rho ; 1 / u)}, \quad|u|=1 .
$$

From (41) it follows by using $\gamma(\rho ; 1)=\sigma(\rho ; 1)$ and (23) that

$$
\lim _{\rho \downarrow 0} \frac{d}{d \rho} u_{0}(\rho)=-\lim _{\rho \downarrow 0} \frac{\partial}{\partial \rho} \gamma(\rho ; 1) / \gamma^{\prime}(1)=\frac{\beta_{1}}{(2-a) \gamma^{\prime}(1)} \text {. }
$$

This implies that, cf. (43),

$$
\lim _{\rho \downarrow 0} K(\rho ; 1)=-\left\{\gamma^{\prime}(1)\right\}^{-2} .
$$

Moreover, it follows from (29) and the fact that for $\rho>0$ the conformal mapping $\gamma(\rho ; z)$ is regular at $z=1$, that for every $\delta, 0<\delta<1$, there exists an $M$ independent of $u$ and $\rho$ such that for $|u|=1$ and $\rho$ close to 0 ,

$$
|K(\rho ; u)-K(\rho ; 1)|<M|u-1|^{\delta} .
$$

This implies that by splitting up the second integral in (42) as

$$
\frac{1}{u_{0}^{2}(\rho)-1}\left[\int_{C} K(\rho ; u) \frac{1}{u-u_{0}(\rho)} \frac{d u}{u}-\int_{C} K(\rho ; u) \frac{u_{0}(\rho)}{u u_{0}(\rho)-1} \frac{d u}{u}\right] \text {, }
$$

on both these integrals an extended version of the Sochozki-Plemelj formulas (cf. [11], §16, [1], Lemma I.3.6) may be applied. This leads to

$$
\begin{gathered}
\lim _{\rho \downarrow u} \frac{1}{2 \pi i} \int_{C} K(\rho ; u) \frac{1}{u-u_{0}(\rho)} \frac{d u}{u}=-\frac{1}{2} K(0 ; 1)+\frac{1}{2 \pi i} \int_{C} K(0 ; u) \frac{1}{u-1} \frac{d u}{u}, \\
\lim _{\rho \downarrow 0} \frac{1}{2 \pi i} \int_{C} K(\rho ; u) \frac{u_{0}(\rho)}{u u_{0}(\rho)-1} \frac{d u}{u}=\frac{1}{2} K(0 ; 1)+\frac{1}{2 \pi i} \int_{C} K(0 ; u) \frac{1}{u-1} \frac{d u}{u} .
\end{gathered}
$$

The integrals on the right-hand sides of (48) have to be understood as principle values. Finally, by using, cf. (44), that

$$
\lim _{\rho \downarrow 0} \frac{\alpha \rho}{u_{0}^{2}(\rho)-1}=\frac{2-a}{2 a} \gamma^{\prime}(1)
$$

the assertion for the case $a<2$ follows from (42), (47), (48) and (45).

\section{Asymptotic behaviour of the queueing process}

With the aid of the analysis of the preceding section the main theorem on the ergodic properties of the queueing system described in Section 2 can be formulated. Let $\gamma_{0}(w)$ be the inverse of the conformal mapping $\gamma(z)$.

Theorem 4. The $M / G / 1$ queueing system with two types of customers and 
paired services is transient if $a>2$, it consists of null states if $a=2$, and it is ergodic if $a<2$. Further, in the case $a<2$, for $\left|z_{1}\right| \leqq 1,\left|z_{2}\right| \leqq 1$,

$$
\Psi\left(z_{1}, z_{2}\right):=\lim _{t \rightarrow \infty} E\left\{z_{1}^{y_{1}^{(t)}} z_{2^{y}}^{{ }^{(t)}}\right\}=\psi_{0} \beta\left(\frac{1-\frac{1}{2} z_{1}-\frac{1}{2} z_{2}}{\alpha}\right)
$$

$$
\begin{gathered}
\times\left[1+\frac{\left(1-z_{1}\right)\left(1-z_{2}\right)}{1-\frac{1}{2} z_{1}-\frac{1}{2} z_{2}} \frac{1-\beta\left(\frac{1-\frac{1}{2} z_{1}-\frac{1}{2} z_{2}}{\alpha}\right)}{\beta\left(\frac{1-\frac{1}{2} z_{1}-\frac{1}{2} z_{2}}{\alpha}\right)-z_{1} z_{2}} \frac{1-\gamma_{0}\left(z_{1}\right) \gamma_{0}\left(z_{2}\right)}{\left\{1-\gamma_{0}\left(z_{1}\right)\right\}\left\{1-\gamma_{0}\left(z_{2}\right)\right\}}\right], \\
\psi_{0}=\lim _{t \rightarrow \infty} \operatorname{Pr}\left\{y_{1}(t)=0, y_{2}(t)=0\right\}=\frac{1-\frac{1}{2} a}{1-\frac{1}{2} a+a \gamma^{\prime}(1)} \\
\lim _{t \rightarrow \infty} E\left\{y_{1}(t)\right\}=\lim _{t \rightarrow \infty} E\left\{y_{2}(t)\right\}=\frac{1}{2} a\left[1+\frac{1-\psi_{0}}{1-\frac{1}{2} a} \frac{\beta_{2}}{2 \beta_{1}^{2}}\right] .
\end{gathered}
$$

Proof. If is easy to see that for the queueing process defined in Section 2 each state in the space $\{0,1,2, \cdots\} \times\{0,1,2, \cdots\}$ has the same classification, and that this process is aperiodic. In the case $a>2$ it follows from Theorem 2, (15) and (2) that

$$
\int_{0}^{\infty} \operatorname{Pr}\left\{y_{1}(t)=0, y_{2}(t)=0\right\} d t<\infty
$$

so that the process is transient. In the case $a=2$, Theorem 2 and Theorem 3 imply respectively, cf. (15), (16),

$$
\begin{gathered}
\int_{0}^{\infty} \operatorname{Pr}\left\{\boldsymbol{y}_{1}(t)=0, \boldsymbol{y}_{2}(t)=0\right\} d t=\infty \\
\lim _{t \rightarrow \infty} \operatorname{Pr}\left\{\boldsymbol{y}_{1}(t)=0, \boldsymbol{y}_{2}(t)=0\right\}=0
\end{gathered}
$$

so that the queueing system consists of null states. Finally, in the case $a<2$ Theorem 3 leads with (16) and (15) to (51), thus showing that the process is ergodic. With the same technique as applied in the proof of Theorem 3 the generating function $\Psi\left(z_{1}, z_{2}\right)$ in (50) can be obtained from (13) and Theorem 1 for $z_{1} \in \Lambda^{+}, z_{2} \in \Lambda^{+}$, and by analytic continuation in the whole region $\left|z_{1}\right| \leqq 1$, $\left|z_{2}\right| \leqq 1$. The moments (52) follow in a standard way from the generating function $\Psi\left(z_{1}, z_{2}\right)$.

Remark 1. In order to obtain the above result it was assumed that $\beta_{3}<\infty$, cf. Section 2. Theorem 4 also holds without this assumption, but the proof becomes more tedious. Because the expansion (22) is not valid if $\beta_{3}=\infty$, more general theorems than Kellogg's theorem have to be applied in order to prove the inequalities (30), cf. [1], Theorem II.8.2, [13], Chapter IX, Part I. 
Next, the relaxation time of the queueing system will be discussed, cf. [3], §III.7.3. The relaxation time $T$ of the probability that the system is empty is by definition the smallest positive value for which it holds that

$$
\operatorname{Pr}\left\{\boldsymbol{y}_{1}(t)=0, \boldsymbol{y}_{2}(t)=0\right\}-\psi_{0}=O\left(e^{-t / T}\right), \quad t \rightarrow \infty .
$$

It is determined by the abscissa of convergence $\rho_{c}$ of the Laplace transform $\Psi(\rho ; 0,0)-\psi_{0} / \rho$, in fact $T=-1 / \rho_{c}$. The discussion will be restricted to the fairly general case that the Laplace-Stieltjes transform $\beta(\zeta)$ has an abscissa of convergence $\zeta_{c}<0$, and that $\beta(\zeta) \uparrow \infty$ as $\zeta \downarrow \zeta_{c}$. In this case the function $\sigma(\rho ; u)$, $u \leqq 1$, cf. Lemma 1 , can be continued analytically to the region $\operatorname{Re} \rho>\rho_{0}$; here $\rho_{0}$ is the largest real value for which Equation (18) has a double root. By considering the functions $\sigma^{2}$ and $\beta(\rho+(1-\sigma u) / \alpha)$ for real values of $\sigma$, cf. (19), it is not difficult to see that this largest value $\rho_{0}$ is attained for $u=1$. Hence $\rho_{0}$ is the largest real value for which there exists a $\sigma_{0}$ such that

$$
\sigma_{0}^{2}=\beta\left(\rho_{0}+\frac{1-\sigma_{0}}{\alpha}\right), \quad 2 \sigma_{0}=\frac{-1}{\alpha} \beta^{\prime}\left(\rho_{0}+\frac{1-\sigma_{0}}{\alpha}\right) .
$$

Note that $\sigma_{0}>1$ if $a<2$, that $\sigma_{0}<1$ if $a>2$, and that $\sigma_{0}=1, \rho_{0}=0$ in the case $a=2$. The above implies that the contour $\Lambda(\rho)$ and the conformal mapping $\gamma(\rho ; z),|z| \leqq 1$, can be continued over the interval $\rho_{0} \leqq \rho \leqq 0$. As in Lemma 2 it can be proved that $\Lambda(\rho)$ possesses a tangent at every point, except in the case $\rho=\rho_{0}$ at $w=\sigma_{0}$. Because $|\gamma(\rho ; z)| \leqq \sigma_{0}<1$ for $\rho \geqq \rho_{0},|z| \leqq 1$, in the case $a>2$, relation (15) can be continued over the interval $\rho_{0}<\rho \leqq 0$. This implies that $\rho_{c}=\rho_{0}$ if $a>2$. In the case $a<2$, when $\sigma_{0}>1$, the analytic continuation of the function $\Psi(\rho ; 0,0)$ is more difficult to obtain.

Lemma 4. In the case $a<2$, for $\rho<0$,

$$
\Psi(\rho ; 0,0)=\frac{\frac{\alpha}{2 \pi i} \int \frac{1}{|1-\gamma(\rho ; u)|^{2}} \frac{d u}{u}+\frac{2 \alpha}{u_{0}(\rho) \gamma^{\prime}\left(\rho ; u_{0}(\rho)\right)\{1-\nu(\rho)\}}}{1+\frac{\alpha \rho}{2 \pi i} \int_{C} \sqrt{|1-\gamma(\rho ; u)|^{2}} \frac{d u}{u}+\frac{2 \alpha \rho}{u_{0}(\rho) \gamma^{\prime}\left(\rho ; u_{0}(\rho)\right)\{1-\nu(\rho)\}}},
$$

here $u_{0}(\rho)<1$ is defined by $\gamma\left(\rho ; u_{0}(\rho)\right)=1$ and $\nu=\nu(\rho)$ is the smallest positive root of the equation

$$
\nu=\beta\left(\rho+\frac{1-\nu}{2 \alpha}\right)
$$

Proof. See the appendix.

From (55) it follows that in the case $a<2$ the abscissa of convergence $\rho_{c}$ of $\Psi(\rho ; 0,0)$ is equal to the maximum of $\rho_{0}$, cf. (54), and of the largest branch point $\rho_{1}$ of the function $\nu(\rho)$. 
Theorem 5. The relaxation time $T$ of the $M / G / 1$ queueing system with two types of customers and paired services is equal to $-1 / \rho_{1}$ if $a<2$, to $-1 / \rho_{0}$ if $a>2$, and it is infinite if $a=2$.

Proof. The cases $a=2$ and $a>2$ were completely discussed above. In the case $a<2$ the maximum of $\rho_{0}$ and $\rho_{1}$ has to be determined. By substituting $\nu=2 \sigma-1$ Equation (56) can be written in the form

$$
\sigma=\frac{1}{2}\left[\beta\left(\rho+\frac{1-\sigma}{\alpha}\right)+1\right] .
$$

By using the inequality $\frac{1}{2}(x+1)>\sqrt{ } x$, which holds for $x>0, x \neq 1$, and the monotonicity of the Laplace-Stieltjes transform $\beta(\zeta), \zeta>\zeta_{c}$, it follows readily from (54) and (57) that $\rho_{1}>\rho_{0}$, so that $T=-1 / \rho_{1}$ if $a<2$.

Remark 2. In the case $a<2$ the relaxation time $T$ is equal to the relaxation time of a common $M / G / 1$ system with mean interarrival time $2 \alpha$ and servicetime distribution $B(\tau)$, cf. (56) and [3], §III.7.3.

Example. In the case of an Erlang service-time distribution with $k$ phases $(k=1,2, \cdots) \rho_{0}$ and $\rho_{1}$ can be easily calculated:

$$
\begin{aligned}
& \rho_{0}=\frac{-1}{\beta_{1}}\left\{k+a-(2+k)\left(\frac{1}{2} a\right)^{2 /(2+k)}\right\}, \\
& \rho_{1}=\frac{-1}{\beta_{1}}\left\{k+\frac{1}{2} a-(1+k)\left(\frac{1}{2} a\right)^{1 /(1+k)}\right\} .
\end{aligned}
$$

\section{Additional results}

In order to obtain numerical values for the stationary distribution of the queue-length process the conformal mapping $\gamma(z)$ or its inverse $\gamma_{0}(w)$ has to be determined. In [4], Part IV, and in [1], §IV.3, it is shown how the conformal mapping $\gamma(z)$ and its moments can be determined in general by a numerical procedure. In two cases, that of a negative exponential and that of a degenerate service-time distribution, an explicit expression for the inverse $\gamma_{0}(w)$ has been found from (7). When the service times are negative exponentially distributed

$$
\begin{gathered}
\gamma_{0}(w)=1-\frac{2 \delta}{w} \frac{(1-w)^{2}}{(1-\delta)^{2}} \frac{1-\frac{1}{2} a w}{1-\frac{1}{2} a \delta}\left[1+\frac{1}{\delta} \frac{w-\delta}{1-w} \sqrt{\frac{1-\frac{1}{2} a w \delta^{2}}{1-\frac{1}{2} a w}}\right], \\
\psi_{0}=\frac{\left(1-\frac{1}{2} a\right) \sqrt{\left(1-\frac{1}{2} a\right)\left(1-\frac{1}{2} a \delta^{2}\right)}}{\frac{1}{2} a(1-\delta)\left(1-\frac{1}{2} a \delta\right)+\left(1-\frac{1}{2} a\right) \sqrt{\left(1-\frac{1}{2} a\right)\left(1-\frac{1}{2} a \delta^{2}\right)}}
\end{gathered}
$$

here $\delta:=\sigma(-1)=\{1-\sqrt{1+4 a}\} / 2 a$, cf. (18), (20). When the service times are constant 


$$
\gamma_{0}(w)=w e^{\frac{1}{2} a(1-w)}, \quad \psi_{0}=\frac{\left(1-\frac{1}{2} a\right)^{2}}{1+\frac{1}{4} a^{2}} .
$$

In these two cases $\omega$ is rational $\left(\frac{2}{3}, \frac{1}{2}\right)$, cf. Lemma 2 . In general $\omega$ is not rational, which makes it hard to find explicit expressions for $\gamma_{0}(w)$.

In [1], §II.8, the asymptotic behaviour of the embedded process $\left(\boldsymbol{x}_{1}(n), \boldsymbol{x}_{2}(n)\right)$, cf. Sections 2, 3, as $n \rightarrow \infty$, has been studied. The main result is that if $a<2$,

$$
\begin{gathered}
\lim _{n \rightarrow \infty} E\left\{z_{1}^{x_{1}^{(n)}} z_{2}^{\left.x_{2}^{(n)}\right\}}\right. \\
=\phi_{0} \frac{\left(1-z_{1}\right)\left(1-z_{2}\right) \beta\left(\frac{1-\frac{1}{2} z_{1}-\frac{1}{2} z_{2}}{\alpha}\right)}{\beta\left(\frac{1-\frac{1}{2} z_{1}-\frac{1}{2} z_{2}}{\alpha}\right)-z_{1} z_{2}} \frac{1-\gamma_{0}\left(z_{1}\right) \gamma_{0}\left(z_{2}\right)}{\left\{1-\gamma_{0}\left(z_{1}\right)\right\}\left\{1-\gamma_{0}\left(z_{2}\right)\right\}}, \\
\phi_{0}:=\lim _{n \rightarrow \infty} \operatorname{Pr}\left\{x_{1}(n)=0, x_{2}(n)=0\right\}=\frac{1-\frac{1}{2} a}{\gamma^{\prime}(1)} .
\end{gathered}
$$

Note the difference between the limiting distribution of the embedded process and that of the continuous-time process, cf. Theorem 4. From (51) and (58) the stationary waiting-time distribution can be derived. Clearly, this distribution is the same for both types of customer. Let $\boldsymbol{w}$ be the waiting time for an arriving customer. Because the arrival process is a Poisson process, it follows that $\operatorname{Pr}\{\boldsymbol{w}=0\}=\psi_{0}$. Further, for $j=1,2$, the number of type $j$ customers left behind in the queue by a type $j$ customer at the instant at which his service commences is equal to the number of type $j$ customers that arrived during his waiting time, cf. [3], p. 256. Let $\boldsymbol{x}$ be a random variable with distribution the limiting marginal distribution $(n \rightarrow \infty)$ of $\boldsymbol{x}_{j}(n), j=1,2$. Then application of the foregoing argument at departure instants leads to: for $|z| \leqq 1$,

$$
E\left\{z^{x-1} \mid \boldsymbol{x}>0\right\}=E\left\{\left(\frac{1-z}{2 \alpha}\right)^{w} \mid \boldsymbol{w}>0\right\}
$$

With (58) the above leads to: in the case $a<2$, for $\operatorname{Re} \zeta \geqq 0$,

(60) $E\left\{e^{-\zeta \boldsymbol{\omega}}\right\}=\psi_{0}+\frac{1-\psi_{0}}{\frac{1}{2} a}\left(1-\frac{1}{2} a\right) \frac{1-\beta(\zeta)}{\beta(\zeta)-1+2 \alpha \zeta}, \quad E\{\boldsymbol{w}\}=\frac{1-\psi_{0}}{1-\frac{1}{2} a} \frac{\beta_{2}}{2 \beta_{1}}$.

The model as described in Section 2 can be generalized by the assumption that an arriving customer is with probability $c_{j}$ of type $j, j=1,2, c_{1}+c_{2}=1$. For the asymmetric case the analysis is somewhat simpler, cf. [1]. The asymptotic analysis leads to the following main result: the asymmetric system is ergodic iff $\max \left\{c_{1}, c_{2}\right\} a<1$, and in the ergodic case the relaxation time is equal to that of common $M / G / 1$ system with mean interarrival time $\alpha / \max \left\{c_{1}, c_{2}\right\}$ and servicetime distribution $B(\tau)$, cf. Remark 2 in Section 6 . 


\section{Appendix}

Proof of Lemma 2. Because the root $\sigma(\rho ; u)$ of Equation (18) is simple, except in the case $a=2$ for $\rho=0, u=1$, it follows with (25) and (26) that the contours $\Lambda(\rho), \rho>0$, and $\Lambda$ possess a tangent at every point, with the possible exception for $\Lambda$ at $w=1$ when $a=2$. In this case it follows from (22) that

$$
\begin{gathered}
\lim _{\theta \uparrow^{0}} \frac{d}{d \theta} \cos \theta \sigma(\cos \theta)=-\lim _{\theta \downarrow 0} \frac{d}{d \theta} \cos \theta \sigma(\cos \theta)=\left\{2 \beta_{2} / \beta_{1}^{2}-1\right\}^{-1 / 2}, \\
\lim _{\theta \uparrow^{0}} \frac{d}{d \theta} \sin \theta \sigma(\cos \theta)=\lim _{\theta \downarrow 0} \frac{d}{d \theta} \sin \theta \sigma(\cos \theta)=1 .
\end{gathered}
$$

Hence, the left-hand and right-hand tangents at $w=1$ have different directions, with inner angle $\omega \pi$, cf. (27); $\frac{1}{2} \leqq \omega<1$ since in general $\beta_{2} \geqq \beta_{1}^{2}$.

Proof of Lemma 3. First consider the case $a \neq 2, \rho=0$. Let $w(s)$ be the parametric equation of $\Lambda$ with as parameter its arc length $s$ measured from the point $w=-\sigma(-1)$, cf. (26). By using the properties of the function $\sigma(u)$ given in Lemma 1 it is straightforward to show (for details see [1], Theorem II.8.2) that there exists a constant $M_{1}$ such that for every $s_{1}, s_{2}, 0 \leqq s_{1} \leqq s_{2} \leqq s_{0}\left(s_{0}\right.$ is the length of $\Lambda$ ),

$$
\left|w^{\prime}\left(s_{1}\right)-w^{\prime}\left(s_{2}\right)\right|<M_{1}\left|s_{1}-s_{2}\right| .
$$

By Kellogg's theorem, cf. [13], Theorem IX.7, this inequality implies that $\gamma^{\prime}(z)$ exists and is non-vanishing for $|z| \leqq 1$, and that for every $\delta, 0<\delta<1$, there exists a constant $M_{2}$ such that for every $\theta_{1}, \theta_{2},-\pi \leqq \theta_{1} \leqq \theta_{2} \leqq \pi$,

$$
\left|\gamma^{\prime}\left(\exp \left(i \theta_{1}\right)\right)-\gamma^{\prime}\left(\exp \left(i \theta_{2}\right)\right)\right|<M_{2}\left|\theta_{1}-\theta_{2}\right|^{1-\delta} \text {. }
$$

By a theorem of Hardy and Littlewood, cf. [7], §IX.5, Satz 4, it then follows that for some constant $M_{3}$ for every $z,|z| \leqq 1$,

$$
\left|\gamma^{\prime}(z)-\gamma^{\prime}(1)\right|<M_{3}|1-z|^{1-\delta} \text {. }
$$

This inequality implies (29). A similar argument shows that $(\partial / \partial z) \gamma(\rho ; z), \rho>0$, exists and does not vanish for $|z| \leqq 1$, for every traffic intensity $a$. Next consider the case $a=2, \rho=0$. Because the contour $\Lambda$ has a corner point at $w=1$ with inner angle $\omega \pi$, cf. Lemma 2 , the region $\Lambda^{+} \cup \Lambda$ is mapped conformally onto a region $X^{+} \cup X$ by

$$
\xi(w)=1-(1-w)^{1 / \omega}, \quad \xi(0)=0 .
$$

Then the contour $X$ possesses a tangent at every point. Let $f(z)$ be the conformal mapping of the region $|z| \leqq 1$ onto $X^{+} \cup X$ with $f(0)=0, f^{\prime}(0)>0$. Similarly to the above, it can be shown with the aid of (22) that $f^{\prime}(z)$ exists and is non-vanishing for $|z| \leqq 1$. Further, by the uniqueness of conformal mapping, 


$$
\gamma(z)=1-[1-f(z)]^{\omega}, \quad|z| \leqq 1
$$

Hence, the existence of $f^{\prime}(1) \neq 0$ and the inequalities $\frac{1}{2} \leqq \omega<1$, cf. (27), imply (30). Further, the existence of $f^{\prime}(z) \neq 0,|z|=1, z \neq 1$, implies the existence of $\gamma^{\prime}(z) \neq 0,|z|=1, z \neq 1$.

Proof of Lemma 4. Let $a<2$ and consider the first integral in (47). As $\rho \downarrow 0$, $u_{0}(\rho) \downarrow 1$ and the integral becomes singular, cf. (48). Because $\gamma(z)$ is regular at $z=1$, the latter can be avoided by deforming the path of integration for small values of $\rho$ to a contour $C_{1}$ which includes the points $z=1$ and $z=u_{0}(\rho)$ in its interior. Then, for $\rho$ close to 0 ,

$$
\frac{1}{2 \pi i} \int_{C} \frac{K(\rho ; u)}{u-u_{0}(\rho)} \frac{d u}{u}=\frac{1}{2 \pi i} \int_{C_{1}} \frac{K(\rho ; u)}{u-u_{0}(\rho)} \frac{d u}{u}-\frac{K\left(\rho ; u_{0}(\rho)\right)}{u_{0}(\rho)}
$$

The integral on the right-hand side can be continued to negative values of $\rho$ without encountering any singularities until $\rho_{0}$. For $\rho_{0}<\rho<0$ the integral over $C_{1}$ is equal to that over $C$. The second integral in (47) can be treated similarly. This implies (55) with $\nu(\rho):=\gamma\left(\rho ; 1 / u_{0}(\rho)\right)$, cf. (42), (47), and (43) which implies

$$
K\left(\rho ; u_{0}(\rho)\right)=\frac{-1}{\gamma^{\prime}\left(\rho ; u_{0}(\rho)\right)} \frac{u_{0}^{2}(\rho)-1}{1-\gamma\left(\rho ; 1 / u_{0}(\rho)\right)} .
$$

Since $\gamma(\rho ; u) \in \Lambda(\rho)$ for $|u|=1$, it follows from (7), cf. (14), (10), that

$$
\gamma(\rho ; u) \gamma(\rho ; 1 / u)=\beta\left(\rho+\frac{1-\frac{1}{2} \gamma(\rho ; u)-\frac{1}{2} \gamma(\rho ; 1 / u)}{\alpha}\right), \quad|u|=1 .
$$

Because $\gamma(\rho ; u)$ is regular for $|u|=1$ (cf. the proof of Theorem 3), this relation also holds in some strip $1-\varepsilon \leqq|u| \leqq 1+\varepsilon$. By substituting $u=u_{0}(\rho)$ into the above relation and by using $\gamma\left(\rho ; u_{0}(\rho)\right)=1$ it follows that $\gamma\left(\rho ; 1 / u_{0}(\rho)\right)$ satisfies Equation (56), and the proof of (55) is ready.

\section{Acknowledgement}

The author wishes to thank Professor J. W. Cohen for useful advice during the preparation of this paper.

\section{References}

[1] Blanc, J. P. C. (1982) Applications of the Theory of Boundary Value Problems in the Analysis of a Queueing Model with Paired Services. Mathematical Center Tract 153, Amsterdam.

[2] Blanc, J. P. C. (1985) The relaxation time of two queueing systems in series. Stochastic Models. To appear.

[3] COHEn, J. W. (1982) The Single Server Queue, 2nd edn. North-Holland, Amsterdam.

[4] Conen, J. W. And Boxma, O. J. (1983) Boundary Value Problems in Queueing System Analysis. North-Holland, Amsterdam. 
[5] Fayolle, G. AND IASNOGorodski, R. (1979) Two coupled processors: the reduction to a Riemann-Hilbert problem. Z. Wahrscheinlichkeitsth. 47, 325-351.

[6] Fayolle, G., King, P. J. B. And Mitrani, I. (1982) The solution of certain two-dimensional Markov models. Adv. Appl. Prob. 14, 295-308.

[7] Golusin, G. M. (1957) Geometrische Funktionentheorie. V. E. B. Deutscher Verlag der Wissenschaft, Berlin.

[8] JACKSON, J. R. (1957) Networks of waiting lines. Operat. Res. 5, 518-521.

[9] Kleinrock, L. (1976) Queueing Systems II, Computer Applications. Wiley, New York.

[10] Markushevich, A. I. (1977) Theory of Functions of a Complex Variable, Vol. 3, 2nd edn. Chelsea, New York.

[11] Muschelischwili, N. I. (1965) Singuläre Integralgleichungen. Akademie-Verlag, Berlin.

[12] Nehari, Z. (1952) Conformal Mapping. McGraw-Hill, New York.

[13] Tsusi, M. (1959) Potential Theory in Modern Function Theory. Maruzen, Tokyo. 